\title{
A clinical study of the pre-corneal tear film in diabetic and non-diabetic persons- a case control study
}

\author{
Sudha $\mathbf{R}^{1}$, Praveen Kumar K V', \\ ${ }^{1}$ Associate Professor, ${ }^{2}$ Assistant Professor, Dept. of Ophthalmology, Narayana Medical College, Nellore, Andhra Pradesh, India
}

*Corresponding Author:

Email: praveenkumarafmc@gmail.com

\begin{abstract}
Introduction: Several local and systemic disorders predispose to the development of dry eye disease, common among them being diabetes mellitus. This study aims to assess the incidence of dry eye in diabetics and analyse the factors worsening dry eye in these patients.

Materials and Methods: A total of 200 patients were included in the study. The participants were grouped under two groupsdiabetics and non- diabetics group (control). All patients underwent a thorough ophthalmic examination including visual acuity, slit lamp biomicroscopy of anterior segment. Corneal sensation, tear film break up time, schirmers test, flourescein staining, Rose Bengal and lissamine green staining were also done.

Results: Four hundred eyes of two hundred patients were included. There were a total of $86(43 \%)$ males and 114 (57\%) females in the study. The mean age of males was 50.13 years and the mean age of females was 50.27 years. In diabetics, 38 eyes (19\%) had dry eye, out of which 24 eyes (63.15) were outdoor workers and 14 eyes (36.84\%) were indoor workers and in nondiabetics, 14 eyes (7\%) had dry eye out of which 12 eyes $(85.71 \%)$ were of outdoor workers and 2 eyes $(14.28 \%)$ were of indoor workers. Outdoor workers had statistically significant high risk of developing dry eye compared to indoor workers. Out of 52eyes with dry eye, $23(88.46 \%)$ out of the 26 affected individuals were above 50 years of age and $3(11.53 \%)$ were below 50 years of age. TBUT test was positive in 18 eyes (9\%) in non-diabetics and 38 eyes (19\%) in diabetic group. The schirmers values were significantly lower in diabetics compared to non-diabetics.
\end{abstract}

Conclusion: Diabetic patients are at more risk of developing dry eye compared to controls.

Keywords: Diabetes mellitus, Dry eye, Schirmers test.

\section{Introduction}

The importance of the cornea lies in its ability to refract rays of light at the air-cornea interface and to allow passage of these rays into the eye. Corneal transparency is an important prerequisite for vision. Corneal transparency is aided by the presence of a precorneal tear film which keeps the corneal surface moist. The pre-corneal tear film comprises of three layers, an outer lipid layer, a middle aqueous layer, and an inner mucin layer. Tears are produced shortly after birth and their production increases considerably during the neonatal period while excess reflex tearing starts only after four months of age possibly due to the low innervation of the cornea in the very young. ${ }^{1}$ Tears are drained out of the eye, via the nasolacrimal passages, into the inferior meatus of the nose. ${ }^{2}$ Effective spread of the tear film is dependent on the presence of a normal blink reflex, proper contact between the external ocular surface and eyelids, and the presence of normal corneal epithelium. ${ }^{3}$

In the absence of a normal pre-corneal tear film, the cornea tends to dry out and, leading to development of dry eye. Dry eye is a common condition affecting $5 \%$ to $28 \%$ of adults, among the general population globally. ${ }^{4}$ It has been estimated that about 3.23 million women and 1.68 million men greater than 50 years have dry eye..$^{5,6}$

Several local and systemic disorders predispose to the development of dry eye disease, common among them being diabetes mellitus. While diabetic retinopathy is the more commonly known ocular complication of diabetes, diabetic patients have also been found to have symptoms indicative of dry eye such as foreign body and burning sensations, itching, blurred vision, and photophobia, stinging, dryness, ocular fatigue and redness. There is, thus, an implication of a correlation between diabetes mellitus and tear film abnormalities. This study aims to assess the incidence of dry eye in diabetics and analyse the factors associated with dry eye.

\section{Materials and Methods}

The study was a prospective study conducted in the department of Ophthalmology at a tertiary care centre in South India. Ethical committee clearance was obtained from the study. A written informed consent was obtained from all the participants. A total of 200 patients attending the Ophthalmology OPD were included in the study. The participants were grouped under two groups- diabetics and non- diabetics group (control). Patients with any ocular disorder known to produce dry eye, suffering from any systemic diseases, (other than diabetes mellitus), associated with dry eye such as connective tissue disorders (Sjogren's syndrome, rheumatoid arthritis, lupus erythromatosis), any drug treatment which produces dry eye (such as MAO inhibitors, alpha agonists, beta blockers, thiazides, NSAIDs etc), contact lens users, history of any ocular surgery within last 2 years were excluded from the study. Detailed ophthalmic and systemic 
history was obtained from all the patients. All patients underwent a thorough ophthalmic examination including visual acuity, slit lamp biomicroscopy of anterior segment. In addition to determining blood sugar levels, the following tests were performed. Corneal sensation was tested using a wisp of cotton wool brought onto the cornea in order to elicit a blink response. The cornea was tested in four different quadrants in each eye as well as in the centre of the cornea. Tear film break up time was estimated after staining with fluorescein. Schirmers test was done for the quantitate assessment of dry eye. Flourescein staining, Rose Bengal and lissamine green staining was also done and the score recorded to assess the severity of dry eye.

\section{Results}

Four hundred eyes of two hundred patients were examined in this non randomized prospective study. The participants were divided into two groups, group A comprising of 100 non diabetic individuals and the other group (Group B), comprised of one hundred known diabetics participants. Group A was taken as the control group. There were a total of $86(43 \%)$ males and $114(57 \%)$ females in the study. The mean age of males was 50.13 years and the mean age of females was 50.27 years.

The number of females was slightly greater than the number of males in both the group. Of the total of 200 participants, 53\% participants, (70 males and 36 females) had an outdoor occupation while $47 \%$ (16 males and 78 females) followed largely indoor occupations. 54 participants (32\%) (33 males and 21 females) of non-diabetics and 52 participants (26\%) (37 males and 15 females) of diabetics had an outdoor occupation. Those with indoor occupations were 46 participants (23\%) (9 males and 37 females) among non-diabetics and 48 participants $(24 \%)$ ( 7 males and 41 females) among diabetics. In diabetics, 38 eyes (19\%) had dry eye, out of which 24 eyes (63.15) were outdoor workers and 14 eyes (36.84\%) were indoor workers and in non-diabetics, 14 eyes (7\%) had dry eye out of which 12 eyes $(85.71 \%)$ were of outdoor workers and 2 eyes (14.28\%) were of indoor workers. Out door workers had statistically significant high risk of developing dry eye compared to indoor workers. ( $\mathrm{p}=$ 0.006) [Table 1]

The mean duration of diabetes was 6.8years with a range of 2017 years. 51\% (26 males and 25 females) of individuals had a good control of diabetes while $12 \%$ (3 males and 9 females) had poor control of blood sugar levels. Out of 200 eyes of diabetic patients in the study, 36 eyes $(18 \%)$ had evidence of diabetic retinopathy. The severity of the retinopathy varied from mild NPDR in $22(11 \%)$ eyes of four males and seven females, moderate NPDR in $10(5 \%)$ eyes of four males and one female, severe NPDR in two eyes $(1 \%)$ of one female, while only one $(0.5 \%)$ eye of a male showed the presence of proliferative diabetic retinopathy. There was significant correlation between control of blood sugar and severity of diabetic retinopathy $(\mathrm{p}=0.275)$ [Fig. 1]

Dry eye was present in a total of 52 eyes representing $13 \%$ of the eyes in the study. Out of 52eyes with dry eye, $23(88.46 \%)$ out of the 26 affected individuals were above 50 years of age and $3(11.53 \%)$ were below 50 years of age. 7 non diabetics $(7 \%)$ with DED were above 55 years of age, while 19 (19\%) diabetics had developed DED at a younger age.

The symptoms of dry eye disease are non-specific and consist mainly of itching, burning of eyes, and foreign body sensation. $33 \quad(16.5 \%)$ individuals presented with complaints of itching, 31(15.5\%) reported with foreign body sensation and 20(10\%) complained of a burning sensation in the eyes. [Fig. 2]

TBUT test was positive in 18 eyes (9\%) in nondiabetics and 38 eyes (19\%) in diabetic group. Positive Schirmer's tests were seen in 14 eyes $(7 \%)$ of nondiabetics and 48 eyes (24\%) eyes of diabetics. The schirmers values were significantly lower in diabetics compared to non-diabetics $(\mathrm{P}=0.01)$.

Fluorescein staining was positive in 38 eyes (19\%) of diabetic patients as compared to $8(4 \%)$ eyes of nondiabetics. All the eyes showed NEI grading of 3 . Rose Bengal testing is done to detect the presence of dead and devitalized epithelial cells. 6 eyes $(3 \%)$ of nondiabetic group (Group A) as well as 38 eyes (19\%) in group B had grade 3 Rose Bengal staining. Lissamine green staining for detection of mucin deficient conjunctival areas showed grade 3 positive results in 38 eyes (19\%) of Group B individuals. [Table 2]

Table 1: Duration of diabetes and dry eye

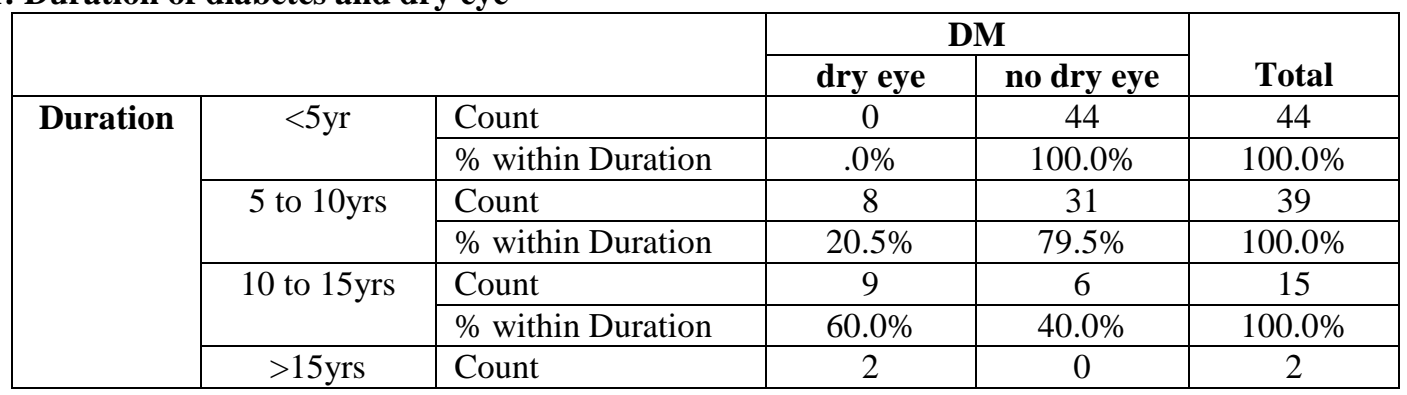




\begin{tabular}{|l|c|l|c|c|c|}
\hline & & $\%$ within Duration & $100.0 \%$ & $.0 \%$ & $100.0 \%$ \\
\hline & Total & Count & 19 & 81 & 100 \\
\hline & & $\%$ within Duration & $19.0 \%$ & $81.0 \%$ & $100.0 \%$ \\
\hline
\end{tabular}

Table 2: Table showing positive vital stains in cases and controls

\begin{tabular}{|l|c|c|c|c|c|c|c|c|c|c|}
\hline \multicolumn{1}{|c|}{ Age group } & 40-45yrs & \multicolumn{2}{c|}{ 46-50yrs } & \multicolumn{2}{c|}{ 51-55yrs } & \multicolumn{2}{c|}{ 56-60yrs } & \multicolumn{2}{|c|}{ 61-65yrs } \\
\hline Test & M & F & M & F & M & F & M & F & M & F \\
\hline TBUT & & & & & 1 & & 3 & 1 & 3 & 1 \\
\hline Schirmer's test I & & & & & 1 & & 2 & 1 & 2 & 1 \\
\hline Fluorescein staining & & & & & & & 1 & 1 & 2 & \\
\hline Rose Bengal staining & & & & & & & 1 & & 2 & \\
\hline Lissamine green staining & & & & & & & 1 & & 2 & \\
\hline
\end{tabular}

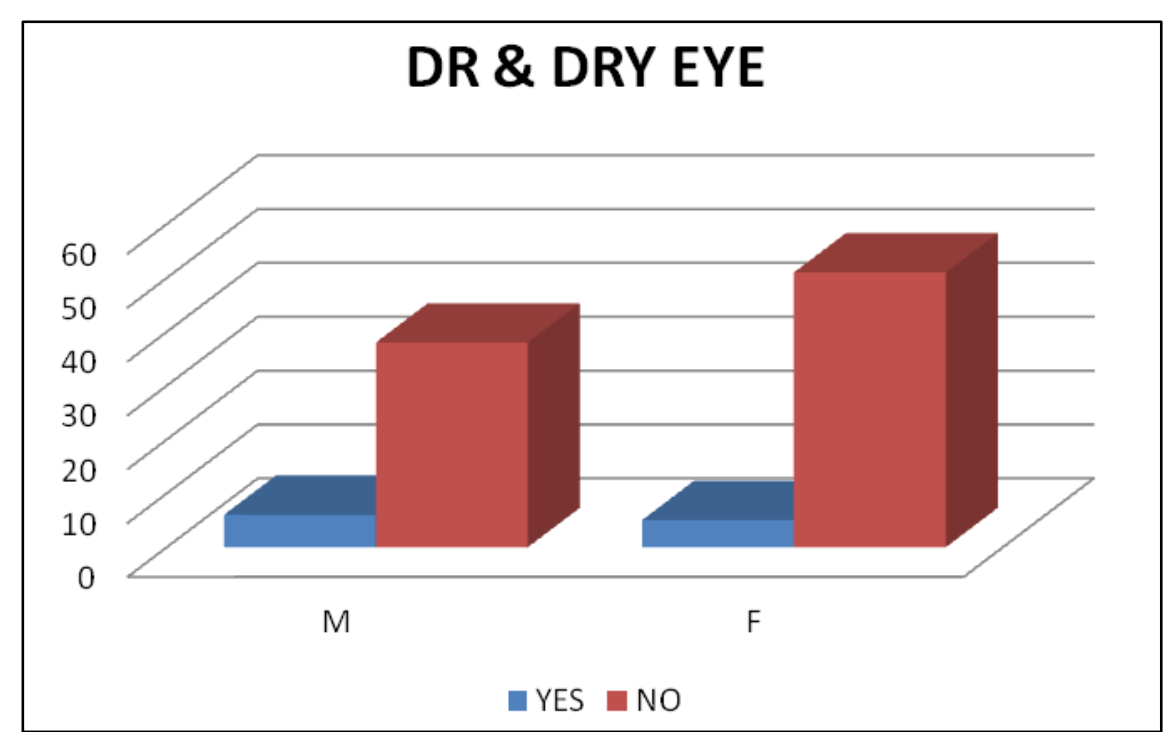

Fig. 1: Showing relation between diabetic retinopathy and dry eye

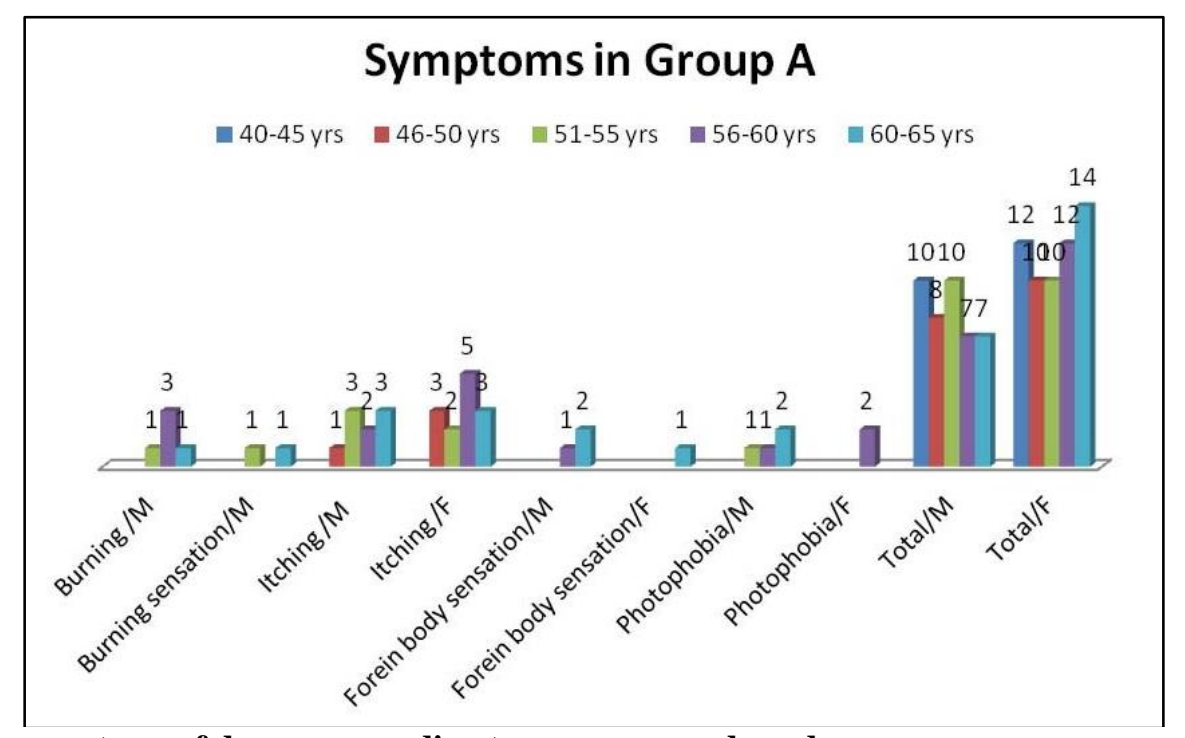

Fig. 2: Showing symptoms of dry eye according to age group and gender 


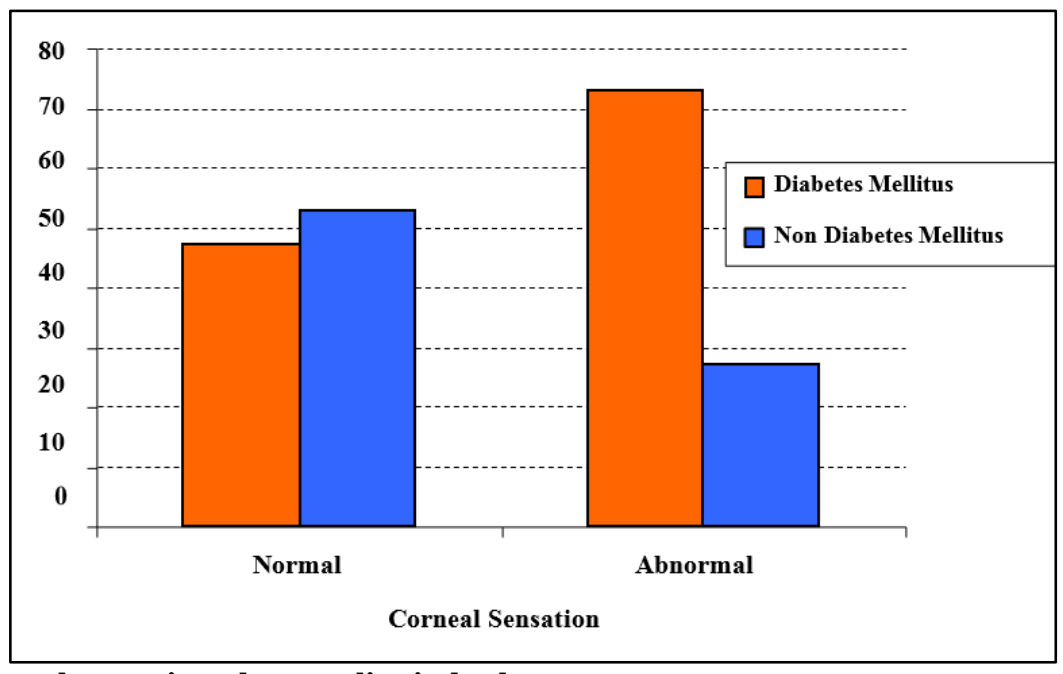

Fig. 3: Showing corneal sensation abnormality in both groups

\section{Discussion}

This hospital based study was done on 400 eyes of 100 normal individuals (Group A) and 100 diabetics (Group B). There were a total of 86 males and 114 females in the study. The youngest participant was a female aged 40 years and the oldest was a 65 years old male. The mean age of all participants was 50.31 years. The mean age of the men was 50.13 years and the mean age of the women was 50.27 years. Of the total of 200 participants, 53\% participants, (70 males and 36 females) had an outdoor occupation while $47 \%$ (16 males and 78 females) followed largely indoor occupations. 54 participants (32\%) (33 males and 21 females) of group A and 52 participants (26\%) (37 males and 15 females) of Group B had an outdoor occupation. Those with indoor occupations were 46 participants (23\%) (9 males and 37 females) from Group A and 48 participants (24\%) (7 males and 41 females) from group B. Environmental exposure is thought to play a role in the development of DED. In this series, group A, six (5.66\%) individuals, 3 males \& 3 females, with outdoor occupations had Dry Eye Disease (DED) while in group B 12(11.32\%) individuals, 10 males \& 2 females, had DED. The influence of outdoor occupation on development of DED is statistically significant with $\mathrm{p}=0.006$. Individuals belonging to group B had been suffering from diabetes mellitus for periods varying from 2 years to 17 years. The mean duration of diabetes was 6.8 years. It is well known that diabetic changes in the retina are duration dependent, and diabetics with over ten years of the disease will start to develop retinopathy. The severity of retinopathy is influenced by the state of control of the diabetes.47 51\% (26 males and 25 females) of individuals had a good control of diabetes while $12 \%$ (3 males and 9 females) had poor control of their blood sugar levels. Diabetic retinopathy had affected 36(18\%) eyes of 9 males and 9 females. The severity of the retinopathy varied from mild NPDR in $22(11 \%)$ eyes of four males and seven females, moderate NPDR in $10(5 \%)$ eyes of four males and one female, severe NPDR in two eyes $(1 \%)$ of one female, while only one $(0.5 \%)$ eye of a male showed the presence of proliferative diabetic retinopathy. $0.275 \mathrm{p}$ value indicates that the correlation between control and severity of retinopathy was highly significant. DED is a fairly common clinical entity which tends to affect between $5 \%$ to $28 \%$ of adults among the general population globally. ${ }^{5,6}$ an increased incidence of DED is seen amongst older people. ${ }^{7,8}$ In the present study, DED was found to affect a total of 52 eyes representing $13 \%$ of the eyes in the study. 23 out of the 26 affected individuals were above 50 years of age and 3 were below 50 years of age. Seven non diabetics with DED were above 55 years of age, while 19 diabetics had developed DED at a younger age. Women are affected twice as often as men. ${ }^{5,6}$ Moss and associates. ${ }^{7}$ too, reported a higher incidence of dry eye among females, $16.7 \%$ compared to $11.4 \%$ in males, but this difference tends to occur only in the older age groups. ${ }^{8}$ Dry eye was detected in a total of 15 males and 11 females. In this series no patient was older than 65 years. The prevalence of DED is significantly higher in diabetic individuals, affecting $20 \%$ to $37 \%$ of all diabetics. ${ }^{9}$ Seifart and associates reported that this was due to loss of conjunctival goblet cells, decreased corneal sensitivity and neuropathy involving the lacrimal glands. ${ }^{10}$ The incidence of DED is higher with longer durations of diabetes. ${ }^{11}$ A $19 \%$ prevalence of DED was present in group B diabetic patients and this correlated well with the duration of diabetes. Maximum frequency of occurrence of DED was in 9 individuals whose diabetes had been present between 10-15yrs while 2 diabetics whose duration of diabetes was more than $15 \mathrm{yrs}$ years had DED (100\% occurrence). P value $=0.01$. Along with a higher age related incidence the severity of symptoms is also found to be greater amongst older people. ${ }^{8}$ The symptoms are non specific 
and consist mainly of itching, burning of eyes, and foreign body sensation. $33(16.5 \%)$ individuals presented with complaints of itching, 31(15.5\%) reported with ocular foreign body sensations and $20(10 \%)$ complained of a burning sensation in their eyes. However, it was seen that $7(36.84 \%)$ diabetics had fewer symptoms than their non-diabetic counterparts. It is thought that this may be due to diabetic damage to the corneal nerves leading to a relative corneal anaesthesia ${ }^{12,13}$ None of the group A (non-diabetics) with DED showed any impairment of corneal sensation. Corneal sensation was also detected to be normal in 12 diabetics with DED. [Fig. 3] Normal Tear Film Break up Time (TBUT) is taken to be around 10 seconds. A recent report by Kinoshita et al stated that corneal sensitivity is reduced during phases of hyperglycemia as a result of changes in corneal hydration control. ${ }^{14}$ Reduced corneal sensation was detected in $14(73.1 \%)$ eyes of 7 individuals with DED in group B. with a significant $\mathrm{P}$ value $=0.12$, and the test is deemed to be positive when TBUT is below this value. The longest TBUT recorded in this series was 47 seconds in group A and 35 seconds in group B. with males generally having shorter times (mean 18.54 seconds) than women (mean 22.30 seconds) especially amongst diabetics. TBUT test was positive in 18 eyes (9\%) belonging to the non-diabetic group and 38 eyes (19\%) in the diabetic group. This is consistent with the fact that severity of DED is greater in older individuals. ${ }^{7,8}$ Tear film adequacy as tested by Schirmer's test shows considerably lesser wetting of the strip in DED. ${ }^{15}$ Positive Schirmer's tests were seen in 14 eyes (7\%) of non-diabetics and 48 eyes (24\%) eyes of diabetics There were significantly lower values amongst diabetics with DED $(\mathrm{P}=0.01)$. Fluorescein staining was positive in 38 eyes $(19 \%)$ of diabetic patients as compared to $8(4 \%)$ eyes of non-diabetics. NEI grading of the corneal staining is taken to be positive if the score is 3 or more. All the eyes showed NEI grading of 3. There were no eyes with higher NEI grading. Rose Bengal testing is done to detect the presence of dead and devitalized epithelial cells. 6 eyes (3\%) of non-diabetic group (Group A) as well as 38 eyes $(19 \%)$ in group B had grade 3 Rose Bengal staining. Lissamine green staining for detection of mucin deficient conjunctival areas showed grade 3 positive results in 38 eyes (19\%) of Group B individuals. All these findings strongly suggest that dry eye is a significant feature of the diabetic ocular surface disease. Schirmer's test results were significantly positive in diabetic individuals $(\mathrm{p}=0.01)$ and were also significantly lower in those with poor metabolic control. These results are suggestive of a neuropathy involving the lacrimal gland.

\section{References}

1. Bedden U, Turgut-Coban D, Aygun C, Ulu-Gungor I, Sullu Y, Erkan, et al. Lacrimal secretion in full-term and premature babies. Trans Ophthalmol. 1974;94:283-289.

2. Doane MG. Blinking and the mechanism of the lacrimal drainage system. Ophthalmology. 1981;88(8):844-851.

3. Lutofsky S, Holly FJ. Pre ocular tear film in health, disease and contact lens wear. Ophthalmology. 1986;663:669.

4. Caffery B. "Uncomfortable Eyes" might be an indication of Dry Eye Syndrome. Avaliable from:URL:http://www.jpma.org.pk/full_article_text.php? article_id $=1255$.

5. Schaumberg DA, Debra A, Reza Dana, Julie E, David A, Sullivan, et al. Prevalence of dry eye disease among us men, estimates from the Physicians' Health Studies. Arch Ophthalmology. 2009;127(6):763-768.

6. Debra A, Schaumberg DA, Reza Dana, Julie E, David A. Sullivan, et al. Prevalence of dry eye disease among us women: Am J Ophthalmology. 2003;136(2):318-326

7. Felskis T, Katsanos K, Kitsanou M. Long-term incidence of dry eye in an older population. Optom Vis Sci. 2008;185(8):668-674.

8. Sarah Wild. Global prevalence of diabetes estimates for the year 2000 and projections for 2030. Diabetes Care. 2004;27(5):1047-1053.

9. Kaiserman I, Kaiserman N, Nakar S. Tear secretion and tear film function in insulin diabetic patients. Am J Ophthalmol. 2005;139:498-503.

10. Hill RM. Clinical Approach to Corneal Dystrophies and Metabolic Disorders. Basic and Clinical Science Course. American Academy of Ophthalmology. (2004-2005) Section 8th, 317-338.

11. Janjetovi Z, Vukovi, Arar S, Besli R, Vajzovi, Dalipi V, et al. The dry eye syndrome and diabetes. Ophthalmology. 2001;54:764-773.

12. Ishida N, Rao GN, Del Cerro M, Aquarelle JV. Corneal nerve alterations in diabetes mellitus. Arch Ophthalmol. 1984;102(9):1380-1384.

13. Li J, Mei C, Hu Y. A study on histochemistry and electron microscopy of corneal nerves in experimental diabetic rabbits. Chung Hua Yen KO Tsa Chih. 1996;32(4):258-259. Available from: URL: http://www.ncbi.nlm.nih.gov/pubmed/9590839

14. Kinoshita S, Kiorpes TC, Friend J. Goblet cell density in ocular surface disease, A better indicator than tear mucin. Arch Ophthalmol. 1983;101(8):1284-1287.

15. Goebbels M. Tear secretion and tear film function in insulin dependent diabetics. Br J Ophthalmology. 2000;84(1):19-21.

How to cite this article: Sudha $R$, Praveen Kumar KV. A clinical study of the pre-corneal tear film in diabetic and non-diabetic persons- a case control study. Ind J Clin Exp Ophthalmol. 2018;4(3):401-405. 